DOI:

10.1038/nrn2279

AFFECTIVE DISORDERS

\title{
Resisting stress
}

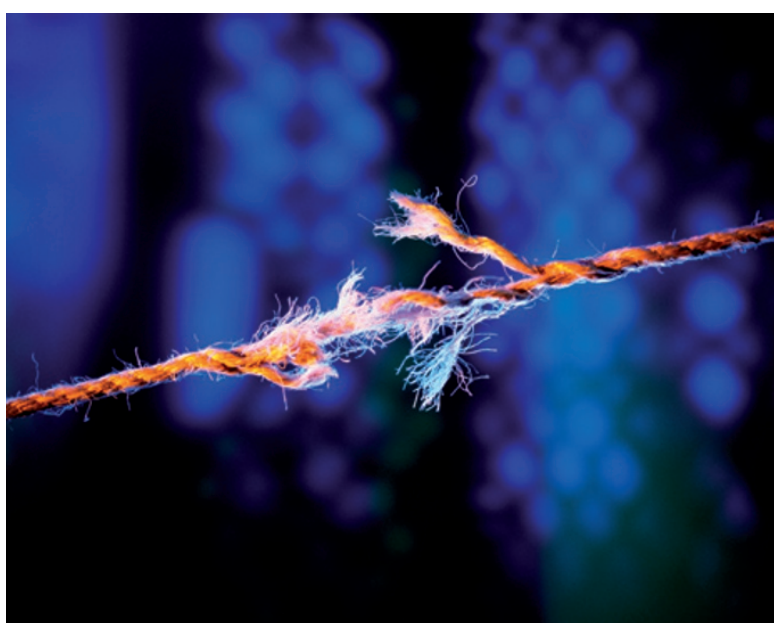

A scientific career brings all kinds of stress: deadlines, competition for positions and grants, having papers rejected... If stress can lead to depression, why aren't we all depressed? According to Nestler and colleagues, the answer might lie partly in adaptations in the mesolimbic dopamine circuit that increase an individual's resilience.

The authors subjected mice to social defeat for 10 days by introducing each animal daily into the cage of an aggressive mouse until the 'intruder' showed submissive behaviour. They then tested the social interaction level of all defeated mice and divided them into two groups: one consisting of 'susceptible' mice that showed reduced social interaction and the other consisting of 'resilient' mice that demonstrated similar interaction to control mice.

Susceptible and resilient mice did not differ in body weight, social interaction or exploration measures before the chronic stress, but afterwards the susceptible animals were lighter and displayed social withdrawal, anhedonia and altered reward processing.

The authors then measured brainderived neurotrophic factor (BDNF) levels in the nucleus accumbens (NAc), which is part of the reward system. Only susceptible mice showed increased BDNF levels and downstream BDNF signalling after chronic stress. Interestingly, injecting BDNF into the NAc reduced resilience, and blocking BDNF signalling increased it, indicating that BDNF induction in the NAc mediates the behavioural effects of chronic social stress.

How does chronic stress increase BDNF levels in susceptible animals? $B d n f$ gene expression in the NAc did not differ between the groups, but knocking down the gene in the ventral tegmentum area (VTA), which projects to the NAc, increased the proportion of resilient mice and attenuated the effects of social defeat, suggesting that the VTA is the source of the elevated NAc BDNF levels.

Social defeat also increased the firing rate of VTA neurons in susceptible mice (but not resilient ones). In addition, decreasing neuronal excitability by overexpressing the $\mathrm{K}^{+}$channel Kir2.1 in the VTA of susceptible mice reduced BDNF levels in the NAc and induced a resilient phenotype; expressing a dominant-negative form of $\mathrm{K}^{+}$channel in resilient mice had the opposite effect.
Microarray analysis of gene expression in the VTA revealed changes in the expression of a larger number of genes in resilient mice than in susceptible ones. Three of the upregulated genes coded for voltage-gated $\mathrm{K}^{+}$channels, leading the authors to speculate that in resilient mice the upregulation of these genes might counteract the defeat-induced excitation of VTA neurons and increased BDNF release into the NAc that is seen in susceptible mice.

The central role of BDNF in creating susceptibility to the effects of social defeat was explored further in mice that were homozygous for the Met or the Val allele of the $B d n f$ gene. Val/Val mice exposed to social defeat had a susceptible phenotype, whereas Met/Met mice were resilient and showed a smaller increase in BDNF levels after stress. Interestingly, the authors also found that BDNF levels in post-mortem NAc samples from depressed patients were $40 \%$ increased compared with those from non-depressed people.

These intriguing findings indentify a mechanism that might underlie resilience to the effects of chronic social stress. An important future challenge is to find predictors of resilience and vulnerability that can be observed before chronic stress takes place.

Leonie Welberg

ORIGINAL RESEARCH PAPER Krishnan, V. et al. Molecular adaptations underlying susceptibility and resistance to social defeat in brain reward regions. Cell 131, 391-404 (2007) 